\title{
TRATAMENTO DO COTOVELO RÍGIDO COM ARTROPLASTIA DE INTERPOSIÇÃO ASSOCIADA AO FIXADOR EXTERNO ARTICULADO
}

\author{
STIFF ELBOW TREATMENT BY INTERPOSING ARTHROPLASTY \\ ASSOCIATED TO HINGED EXTERNAL FIXATOR
}

Alberto Naoki Miyazaki' , Marcelo Fregoneze ${ }^{2}$, Pedro Doneux Santos ${ }^{3}$, Luciana Andrade da Silva³, Nelson Gennaro Junior ${ }^{4}$, Sergio Luiz Checchia ${ }^{5}$

\section{RESUMO}

Objetivo: Avaliar os resultados da técnica de artroplastia de interposição do cotovelo com fascia lata associada ao uso de fixador externo articulado no tratamento do cotovelo rígido. Métodos: Entre 2001 e 2006 foram operados e acompanhados cinco casos de cotovelo rígido pelo Grupo de Ombro e Cotovelo da Faculdade de Ciências Médicas da Santa Casa Misericórdia de São Paulo, tendo como critérios de inclusão: pacientes com arco de movimento do cotovelo menor que o funcional associado a degeneração dessa articulação que não tiveram indicação de prótese total. A idade dos pacientes variou entre 21 e 55 anos (média de 38). Houve predomínio do sexo masculino (quatro casos) e, em todos, o membro dominante foi o operado. Quanto à etiologia, houve dois casos de sequela de artrite infecciosa, um de sequela pós-traumática e dois de artrite reumatoide. $\mathrm{O}$ arco de movimento no período pré-operatório variou em torno de 20 a $30^{\circ}$ de flexoextensão; em dois casos existia contratura fixa em flexão em $30^{\circ}$ e $65^{\circ}$. Os pacientes foram avaliados segundo os critérios da AMA modificados por Bruce. Resultados: O seguimento médio foi de 54 meses. Todos os pacientes obtiveram melhora no índice de Bruce, que no pré-operatório era de 43,5 e foi para 88,2 no pós-operatório. Houve dois casos excelentes, um bom, um regular e um ruim. Conclusão: A artroplastia de interposição com fascia lata associada ao uso de fixador externo dinâmico no cotovelo rígido é uma alternativa viável para pacientes que não têm indicação de artroplastia total de cotovelo.

Descritores - Articulação do cotovelo; Artroplastia; Amplitude de movimento articular; Fixadores externos

\section{ABSTRACT}

Objective: Assess the results of the elbow/ fascia lata interposing arthroplasty technique associated to the use of a hinged external fixator in the treatment of stiff elbow. Methods: Between 2001 and 2006, five cases of stiff elbow were operated and followed up by the Shoulder and Elbow Group of the Santa Casa Misericórdia de São Paulo Medical Sciences School, establishing the following as inclusion criteria: patients with below-functional elbow range of motion associated to degeneration on that joint, for whom total prosthesis had not been indicated. Patients' ages ranged from 21 to 55 years (mean: 38). Male gender was prevalent (four cases), and, in all cases, the dominant side was operated. Concerning etiology, two cases of infectious arthritis sequels, one post-trauma sequel, and two rheumatoid arthritis were found. Preoperative range of motion ranged from $20^{\circ}$ to $30^{\circ}$ of flexion-extension; in two cases, fixed contracture existed in flexion at $30^{\circ}$ and $65^{\circ}$. The patients were assessed according to Bruce-modified AMA criteria. Results: The mean follow up time was 54 months. All patients showed improvement of the Bruce index, which, preoperatively, was 43.5 , increasing to 88.2 postoperatively. We found two excellent cases, one good, one fair, and one poor. Conclusion: Fascia lata interposing arthroplasty associated to the use of a dynamic external fixator on stiff elbows is a feasible alternative for patients not indicated to total elbow arthroplasty.

Keywords - Elbow joint; Arthoplasty; Range of motion, articular; External fixators

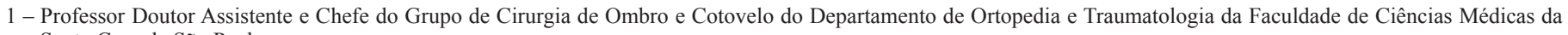
Santa Casa de São Paulo

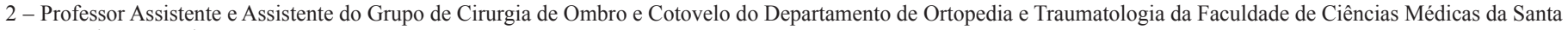
Casa de São Paulo

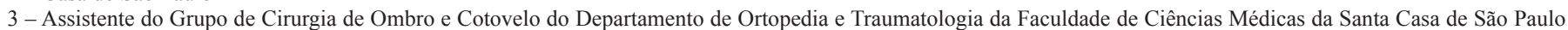

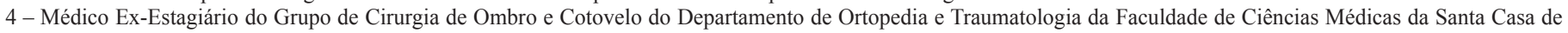
São Paulo

5 - Professor Doutor Adjunto e Chefe de Clínica do Departamento de Ortopedia e Traumatologia da Faculdade de Ciências Médicas da Santa Casa de São Paulo

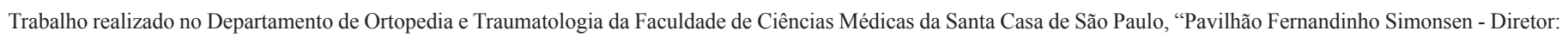
Professor Doutor Osmar Avanzi

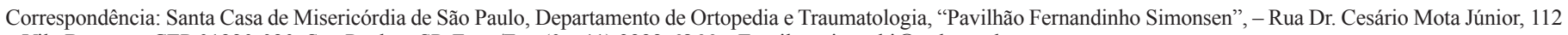

- Vila Buarque. CEP 01220-020. São Paulo - SP. Fone/Fax (0xx11) 3222-6866 - Email: amiyazaki@uol.com.br 


\section{INTRODUÇÃO}

As sequelas de fraturas graves do cotovelo, as artrites reumáticas e infecciosas contribuem para graus variáveis de rigidez dessa articulação ${ }^{(1-3)}$ Segundo Morrey et $a l^{(4)}$, a maioria das atividades realizadas com os membros superiores depende de um arco de movimento do cotovelo de $100^{\circ}$ (arco funcional de Morrey), variando entre 30 e $130^{\circ}$, e de $100^{\circ}$ de pronossupinação. A perda desses graus de movimento gera déficits funcionais, impedindo atividades simples da vida diária, como levar a mão à boca e higiene pessoal, entre outras, além de dor progressiva e incapacitante ${ }^{(5,6)}$.

As primeiras técnicas de artroplastias, tanto de ressecção como de interposição no cotovelo, desenvolveram-se no período entre 1885 e $1947^{(7,8)}$. Após 1947, as artroplastias de substituição com próteses do tipo parcial ou total (restringidas ou dobradiça), fixadas através de cimentação de polimetilmetacrilato $^{(7)}$, foram e continuam sendo uma das principais formas de tratamento de todas as afecções que levam a grande destruição da superfície articular do cotovelo, sejam elas traumáticas ou não ${ }^{(7,8)}$. Embora a artroplastia total ou parcial do cotovelo seja bem difundida e utilizada, não deve ser a opção em pacientes jovens e/ou ativos $^{(2,9-10)}$, nos quais a utilização do membro superior é constante e requer força, pois provoca elevado índice de solturas dos componentes ${ }^{(2,9-10)}$; para esses casos a artrodese seria uma alternativa, mas os pacientes nem sempre a aceitam bem devido à grande limitação do movimento resultante ${ }^{(8,10)}$.

Nos últimos anos, a artroplastia por interposição no cotovelo tem sido resgatada como forma de tratamento da rigidez articular em que a indicação de outras cirurgias, como a artroplastia por substituição ou a artrodese, não consegue suprir todas as necessidades do paciente ${ }^{(6-10)}$.

A interposição com tecidos biológicos como fascia lata e tecido adiposo, para o revestimento de extremidades ósseas, foi introduzida por Murphy em 1902, apud Wright e Sisk ${ }^{(7)}$. Em 1918, Baier, apud Wright e Steward $^{(8)}$, utilizou como tecido de interposição silicone, borracha e membrana à base de cromo, obtendo resultados satisfatórios. No entanto, a fascia lata permanece como o tecido mais comumente utilizado nas artroplastias de interposição, devido à facilidade em ser retirada e por causar menor dano ao sítio doador ${ }^{(1,3,7-8)}$.

A determinação do centro de rotação do capitulo umeral por Steindler ${ }^{(9,11)}$, foi fundamental para o tratamento atual da rigidez de cotovelo. O centro de rotação é o local exato, no plano tridimensional, onde ocorre a flexoextensão do cotovelo, sem que haja alteração do eixo central do braço em relação ao antebraço $0^{(9,11-13)}$.
Com as informações em relação ao centro de rotação, Volkov e Oganesian ${ }^{(6)}$ foram os primeiros autores que associaram o uso do fixador externo em conjunto com interposição da fascia lata, utilizando com sucesso essa técnica em 28 casos de rigidez de cotovelo.

Este trabalho tem como objetivo apresentar os resultados da técnica de artroplastia de interposição do cotovelo com fascia lata associada ao uso do fixador externo articulado no tratamento do cotovelo rígido.

\section{CASUÍSTICA E MÉTODOS}

No período entre outubro de 2001 e julho de 2006, foram operados e acompanhados pelo Grupo de Ombro e Cotovelo do Departamento de Ortopedia e Traumatologia da Faculdade de Ciências Médicas da Santa Casa de Misericórdia de São Paulo - Pavilhão Fernandinho Simonsen - cinco pacientes com cotovelo rígido, nos quais foi utilizada a técnica de artroplastia de interposição com fascia lata associada ao uso do fixador externo articulado no cotovelo.

Utilizamos como critérios de inclusão pacientes que tinham arco de movimento do cotovelo afetado menor que o funcional ${ }^{(4)}$ associado a destruição da superfície articular comprovada por exames de imagem, aliados à contra-indicação de prótese total de cotovelo. Foram excluídos todos os pacientes que não se enquadraram nos critérios estabelecidos acima.

A idade dos pacientes variou entre 21 e 55 anos, com média de 38 anos. Houve prevalência do sexo masculino (quatro casos) e, em todos os casos, o membro dominante foi o operado (Tabela 1).

O tempo médio de evolução dos sintomas, após o inicio do agente causador, foi de seis anos (Tabela 1).

A etiologia das lesões está descrita na Tabela 1.

Em quatro pacientes houve tentativa cirúrgica prévia para ganho da mobilidade articular, sem sucesso. No caso 3 foi realizada a ressecção da cabeça do rádio e, no caso 4, uma artroplastia umeroulnar, isto é, um orifício na fossa olecraniana do distal do úmero ${ }^{(5)}$ (Tabela 1).

A transposição do nervo ulnar foi realizada em três casos (Tabela 1).

O fixador externo dinâmico permaneceu, em média, por 60 dias, sendo retirado após esse período (Tabela 1).

A reconstrução ligamentar do cotovelo foi necessária em um caso em que foi utilizado, como enxerto, o tendão flexor do joelho (grácil) para reparação medial e lateral do cotovelo (Tabela 1).

$\mathrm{O}$ arco de movimento do cotovelo no período préoperatório variou de $20^{\circ}$ a $30^{\circ}$; em dois casos existia contratura fixa em $30^{\circ}$ e $65^{\circ}$ respectivamente (Tabela 2). 
Tabela 1 - Dados dos pacientes

\begin{tabular}{|c|c|c|c|c|c|c|c|c|c|c|c|}
\hline & Paciente & Sexo & $\begin{array}{l}\text { Idade } \\
\text { (anos) }\end{array}$ & Dom. & Etiologia & $\begin{array}{l}\Delta \text { t evolução } \\
\text { (meses) }\end{array}$ & $\begin{array}{c}\Delta \mathrm{t} \text { seguimento } \\
\text { (meses) }\end{array}$ & $\begin{array}{l}\text { Cirurgias } \\
\text { prévias }\end{array}$ & $\begin{array}{l}\text { Ant. } \\
\text { n. ulnar }\end{array}$ & $\begin{array}{l}\Delta \mathrm{t} \text { fixador externo } \\
\text { (meses) }\end{array}$ & Observação \\
\hline 1 & A.R.L. & Masc. & 47 & + & Pós-traum. & 5 & 84 & - & + & 57 & \\
\hline 2 & A.P. & Masc. & 34 & + & Art. infec. & 1 & 72 & 1 & + & 73 & $\begin{array}{l}\text { Reconstrução } \\
\text { ligamentar }\end{array}$ \\
\hline 3 & C.M.F. & Masc. & 55 & + & Art. reum. & 4 & 60 & 1 & - & 54 & \\
\hline 4 & J.M.S. & Masc. & 43 & + & Art. tub. & 17 & 30 & 2 & + & 60 & \\
\hline 5 & J.S.A. & Fem. & 21 & + & ARJ & 5 & 24 & 3 & - & 55 & \\
\hline
\end{tabular}

Fonte: Arquivos (SAME) do Departamento de Ortopedia e Traumatologia da Santa Casa de São Paulo

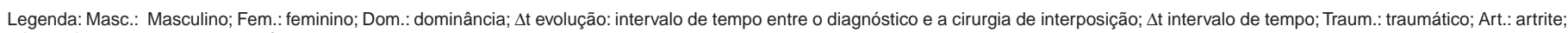
infec.: infecciosa; Reum.: reumatóide; Tub: tuberculosa; ARJ: artrite reumatoide juvenil.

Tabela 2 - Dados dos pacientes

\begin{tabular}{c|c|c|c|c|c|c|c}
\hline & pacientes & $\begin{array}{c}\text { Mob. } \\
\text { Pré op. } \\
\text { F E }\end{array}$ & $\begin{array}{c}\text { Mob. } \\
\text { intra op. } \\
\text { P S S }\end{array}$ & $\begin{array}{c}\text { Bruce } \\
\text { Pré op. }\end{array}$ & $\begin{array}{c}\Delta t \text { pós } \\
\text { op. } \\
\text { (anos) }\end{array}$ & $\begin{array}{c}\text { Mob. } \\
\text { atual } \\
\text { F E }\end{array}$ & $\begin{array}{c}\text { Bruce } \\
\text { Pós op. }\end{array}$ \\
\hline 1 & A.R.L. & $90^{\circ},-70^{\circ}$ & $140^{\circ},-10^{\circ}$ & 43 & 6 & $140^{\circ},-40^{\circ}$ & 92 \\
\hline 2 & A.P. & $65^{\circ},-65^{\circ}$ & $90^{\circ},-5^{\circ}$ & 40 & 5 & $130^{\circ},-35^{\circ}$ & 86 \\
\hline 3 & C.M.F. & $90^{\circ},-60^{\circ}$ & $140^{\circ}, 0^{\circ}$ & 50 & 4 & $110^{\circ},-10^{\circ}$ & 96 \\
\hline 4 & J.M.S. & $70^{\circ},-70^{\circ}$ & $140^{\circ}, 0^{\circ}$ & 40 & 2 & $90^{\circ},-30^{\circ}$ & 96 \\
\hline 5 & J.S.A. & $90^{\circ},-70^{\circ}$ & $140^{\circ},-30^{\circ}$ & 43 & 1,5 & $90^{\circ},-70^{\circ}$ & 71 \\
\hline & Média & $81^{\circ},-67^{\circ}$ & $130^{\circ},-9^{\circ}$ & 43,5 & 3,7 & $112^{\circ},-37^{\circ}$ & 88,2 \\
\hline
\end{tabular}

Fonte: Arquivos do hospital (SAME)

Legenda: Mob.: mobilidade; pré op.: pré-operatória; pós op.: pós-operatória; $\Delta t$ : intervalo de tempo. F: flexão; E: extensão; P: pronação; S: supinação.

\section{DESCRIÇÃO DA TÉCNICA OPERATÓRIA}

Os pacientes foram operados em decúbito dorsal por uma via de acesso posterior; isolou-se e anteriorizou-se o nervo ulnar, quando este estava em sua localização habitual. Realizou-se capsulectomia anterior e posterior e, então, a luxação do cotovelo. Caso não fosse possível, devido a anquilose ou fibrose muito intensa, realizou-se osteotomia onde deveria ser a interlinha articular. Todo o tecido cicatricial e restos de cartilagem articular foram ressecados com regularização de forma uniforme do terço distal do úmero, tentando simular o contorno do osso normal (Figura 1).

A retirada do enxerto do fascia lata $(15 \times 5 \mathrm{~cm})$ foi feita por uma incisão lateral na coxa de aproximadamente $20 \mathrm{~cm}$ e o fechamento da área doadora foi realizada com a ajuda de uma malha de Marlex ${ }^{\circledR}$. Dobrouse o enxerto em três, ficando com formato aproximado de um quadrado com $5 \mathrm{~cm}$ de largura, sendo colocado cobrindo toda a porção distal do úmero e fixado através de pontos transósseos com fio inabsorvível. Após reduzir a articulação, encontrou-se o centro de rotação do cotovelo, que fica no ponto médio entre a tróclea e o capítulo onde normalmente passa o prolongamento da cortical anterior do distal do úmero, e instalouse o fixador externo articulado em sua porção lateral (Figura 2) $^{(14)}$.

Em todos os casos conseguimos, no intraoperatório, mobilidade mínima de $100^{\circ(4)}$, tanto de flexoextensão quanto de pronossupinação do cotovelo (Tabela 2).
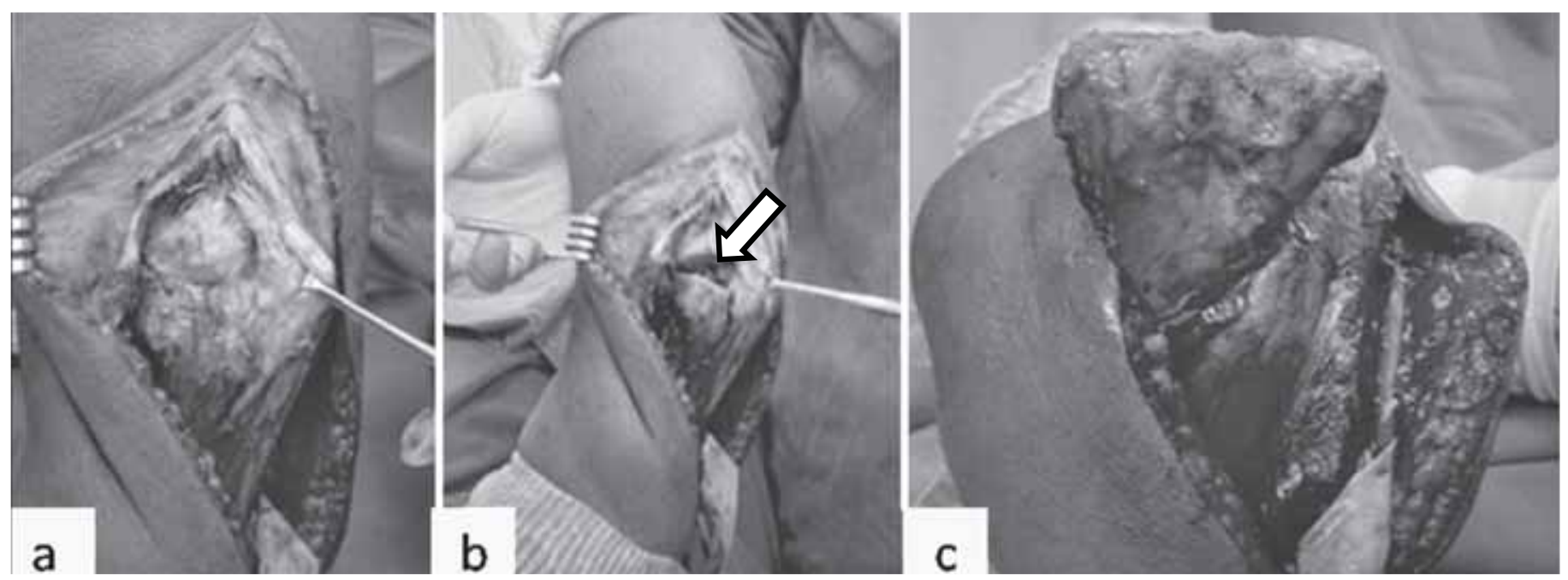

Figura 1 - Imagens intraoperatórias: (a) via de acesso posterior com isolamento do tendão do tríceps, rebatido para lateral;(b) realizada osteotomia no local da interlinha articular (seta) ; (c) visão posterior da região distal do úmero 


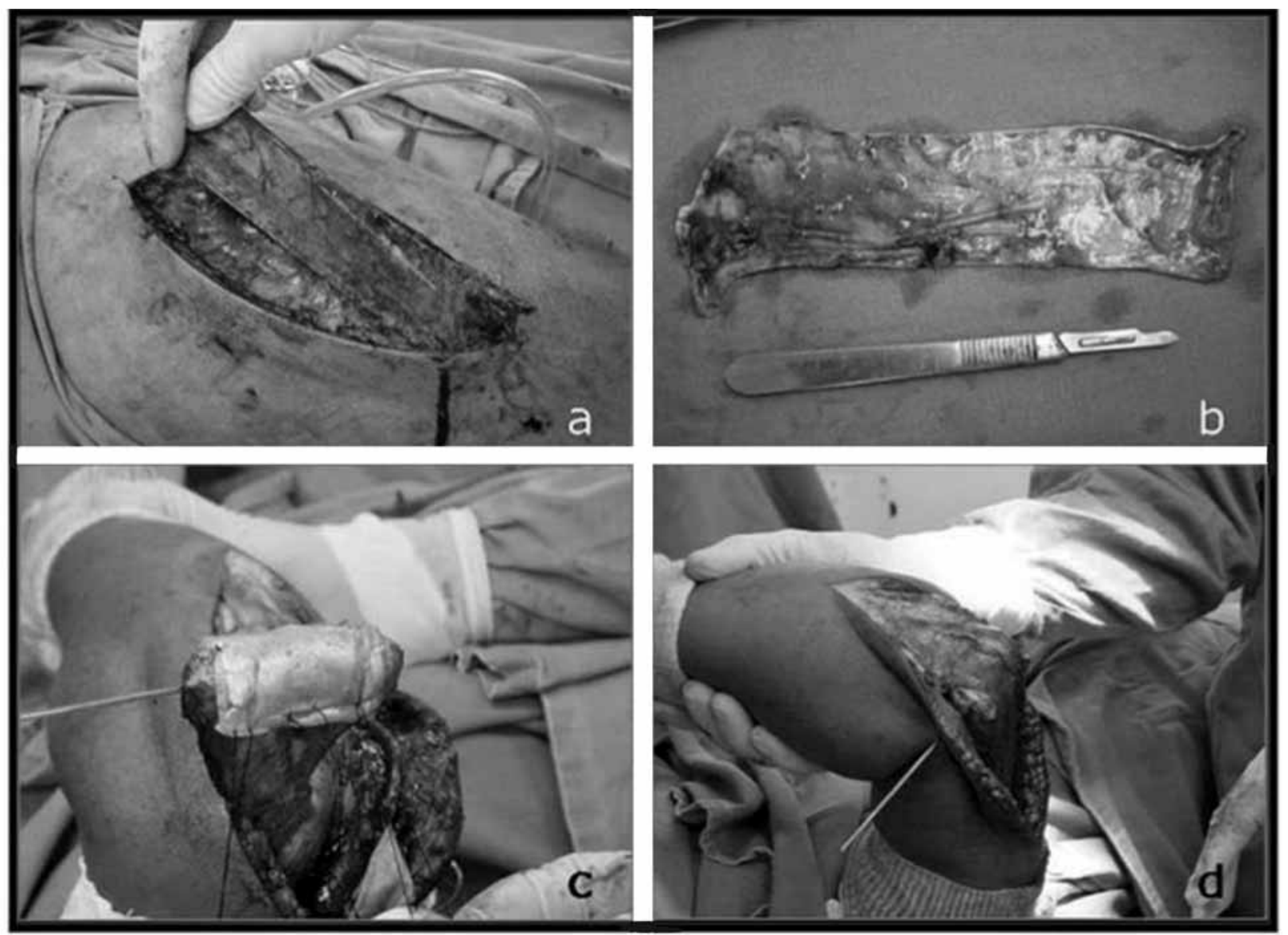

Figura 2 - Imagens intraoperatórias: (a) via de acesso lateral da coxa com isolamento da fascia lata; (b) imagem da fascia lata de \pm 15 por 4,0cm; (c) realizada cobertura da região distal do úmero com a fáscia, presa através de pontos transósseos; (d) visão final com o cotovelo reduzido

Os pacientes foram avaliados pelos critérios da AMA modificados por Bruce et $a l^{(15)}$ quanto à amplitude de movimento no período pós-operatório, às atividades da vida diária e à condição profisssional, além da dor e da anatomia em relação a possíveis deformidades residuais e, por último, foi realizada análise das radiografias.

\section{RESULTADOS}

Os pacientes tiveram seguimento médio de 54 meses, variando entre 24 e 84 meses. Todos obtiveram no período pós-operatório melhora do índice de Bruce et $a l^{(15)}$ (Tabela 2).

Obtivemos dois resultados excelentes (Casos 3 e 4) (Figura 3), um bom (Caso 1), um regular (Caso 2) e um resultado ruim (Caso 5) (Tabela 2).

Houve ganho médio de amplitude articular do cotovelo de $39^{\circ}$ de flexão e $22^{\circ}$ de extensão (Tabela 2 ).

O tempo de seguimento variou entre dois e sete anos, com média de 4,5 anos.

Não tivemos complicações tanto na área doadora (fascia lata) quanto no cotovelo.
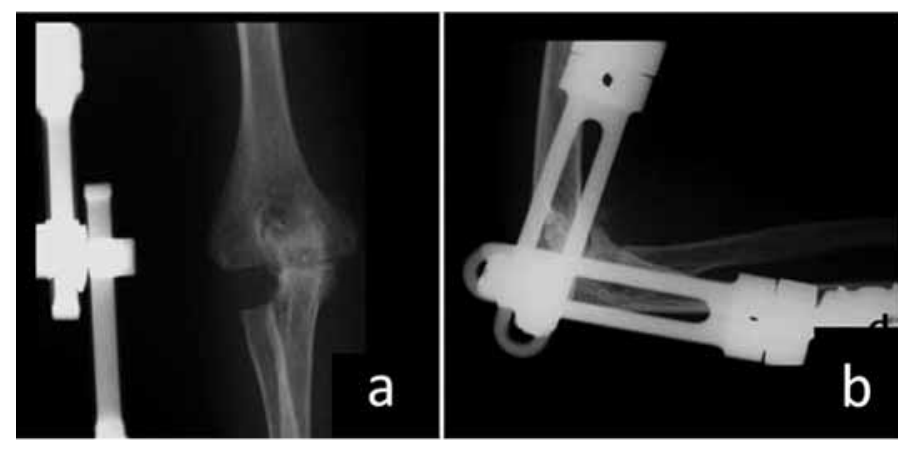

Figura 3 - Caso 4 - Imagens radiográficas do cotovelo no pós-operatório imediato com fixador externo (a) de frente, (b) de perfil

\section{DISCUSSÃO}

A artroplastia de interposição de cotovelo surgiu como uma alternativa para o tratamento de casos graves de limitação dos movimentos do cotovelo, principalmente em pacientes jovens, nos quais a indicação de artroplastia total deveria ser adiada o máximo de tempo possível $^{(2,9,10,16-18)}$. 
Outra alternativa para os jovens seria a artrodese do cotovelo; entretanto, esta acarreta limitação completa e definitiva da função, sobrecarregando as articulações adjacentes; além do mais, a artrodese pode ser realizada na falha de qualquer outro tratamento, mesmo da interposição; deve-se reservá-la como última alternativa ou ainda nos pacientes que necessitam de força no trabalho diário ${ }^{(19)}$.

As causas traumáticas são responsáveis pelas sequelas de rigidez no cotovelo em todos os casos nos trabalhos de Pignatti et al ${ }^{(1)}$, Volkov e Oganesian ${ }^{(6)}$, Cobb e Morrey ${ }^{(19)}$ e Nolla et $a l^{(10)}$, e na maioria dos de Cheng e Morrey $^{(3)}$, enquanto a artrite reumatoide foi a principal causa no trabalho de Ljung et $a^{(2)}$. Em nossa casuística, tivemos dois pacientes com rigidez decorrente de trauma; um evoluiu para artrite infecciosa (Caso 2), um paciente com anquilose por artrite tuberculosa (Caso 4) e dois com artrites inflamatórias (AR, ARJ), evidenciando proporções semelhantes entre as causas traumáticas, inflamatórias e infecciosas de cotovelo rígido. Qualquer análise estatística seria impossível com cinco casos.

Os pacientes que têm como etiologia da rigidez uma doença inflamatória e/ou infecciosa, em geral, são submetidos a diversos procedimentos cirúrgicos prévios $^{(2)}$; o insucesso desses procedimentos antecede a artroplastia de interposição. Podemos observar exatamente esse fato quando avaliamos nossos pacientes, com exceção do caso 1, que foi pós-traumático; os outros foram submetidos a várias intervenções cirúrgicas, como ressecção da cabeça do rádio (Casos 4 e 5), artroplastia umeroulnar (Caso 5) e liberação artroscópica (Casos 4 e 5), na tentativa de ganhar movimento articular.

A idade média do paciente para a realização do procedimento cirúrgico varia conforme o mecanismo de lesão. Assim como na literatura ${ }^{(1,3,8,10-20)}$, nos casos traumáticos normalmente a média é entre a segunda e quarta décadas de vida, e, nas inflamatórias, a partir da sexta década $^{(2-5)}$. Nossa experiência concorda com os padrões descritos acima, com a exceção do caso 5 (21 anos de idade), em que a paciente apresentava artrite reumatoide juvenil (ARJ) e foi submetida a outros três procedimentos prévios (liberação artroscópica do cotovelo, nova liberação com ressecção da cabeça do rádio e uma última tentativa com uma artroplastia umeroulnar), sem sucesso (Tabela 1).

Em relação à técnica cirúrgica, devemos citar que realizamos uma modificação no que foi descrito inicialmente por Froimson ${ }^{(14)}$. Realizamos a cobertura com fascia lata apenas da superfície do úmero, deixando a superfície da ulna descoberta, por acreditarmos que recobrir apenas um dos lados com um enxerto espesso (a fascia lata é dobrada em três partes) é suficiente para manter o espaço articular e facilitar a reabilitação pós- operatória depois da retirada do fixador, facilitando o procedimento cirúrgico (Figura 2).

Observa-se que o ganho de amplitude de movimento articular verificado no intraoperatório é mantido com auxilio do fixador externo dinâmico, assim como descrito por Cobb e Morrey ${ }^{(19)}$, Pignatti et $a^{(1)}$, Cheng e Morrey $^{(3)}$ e Nolla et al ${ }^{(10)}$ (Figura 3). No entanto, esses autores utilizam analgesia pós-operatória, com cateter para bloqueio de plexo braquial por 24 a 48 horas, e fisioterapia com um dispositivo de movimentação contínua passiva (CPM) por mais sete a 10 dias. No nosso serviço, o controle de analgesia é realizado por via endovenosa no período pós-operatório imediato; uma equipe de fisioterapia especializada para acompanhar esses pacientes realiza exercícios pelo menos três vezes ao dia para tentar manter a amplitude de movimento do cotovelo obtida no intraoperatório. O paciente permanece, em média, sete dias hospitalizado.

A motivação do paciente tem sido um fator determinante para o ganho de mobilidade ${ }^{(1,6,9,19-21)}$; em geral, esses pacientes têm restrição total ou quase total da mobilidade articular e já no pós-operatório imediato se consegue um arco de movimento funcional; isso, com certeza, é um grande estímulo para os pacientes continuarem executando os exercícios orientados na fisioterapia. Um dos momentos críticos, na evolução pós-operatória e da motivação do paciente, é o da retirada do fixador externo. Ao retirar o aparelho, observa-se grau variável de instabilidade e perda de força muscular, acompanhada de dor. O fixador externo é quem fornece estabilidade e suporte mecânico para o movimento e a continuidade dos exercícios é fundamental para o sucesso do tratamento $^{(10)}$. Por um período de aproximadamente seis meses a dor diminui de forma progressiva e há ganho gradativo de estabilidade e movimento (Figura 4).

Retiramos o fixador externo, em média, com 60 dias de pós-operatório por acreditamos que nesse período as partes moles já têm um grau de cicatrização capaz de dar estabilidade suficiente para que o paciente inicie seu programa de reabilitação. Cobb e Morrey ${ }^{(19)}$, Morrey ${ }^{(9)}$ e Nolla et $a^{(10)}$ o fazem por volta dos 40 dias, assim como Pignatti et $a^{(1)}$, aos 50 dias.

Complicações como lesão do nervo ulnar (neurites e neuropraxia) são descritas ${ }^{(3,9)}$; entretanto, não tivemos esse tipo de complicação. Fazemos uma cuidadosa dissecção do nervo como um dos primeiros passos da cirurgia e ao final realizamos a transposição anterior do mesmo, quando ele está em sua localização habitual. Muitas vezes, esses pacientes já tiveram o nervo anteriorizado em um procedimento prévio, como nos Casos 1, 2 e 4.

Outra complicação citada $^{(2,9-10)}$ como infecção não ocorreu em nossa casuística. Quanto à instabilidade re- 


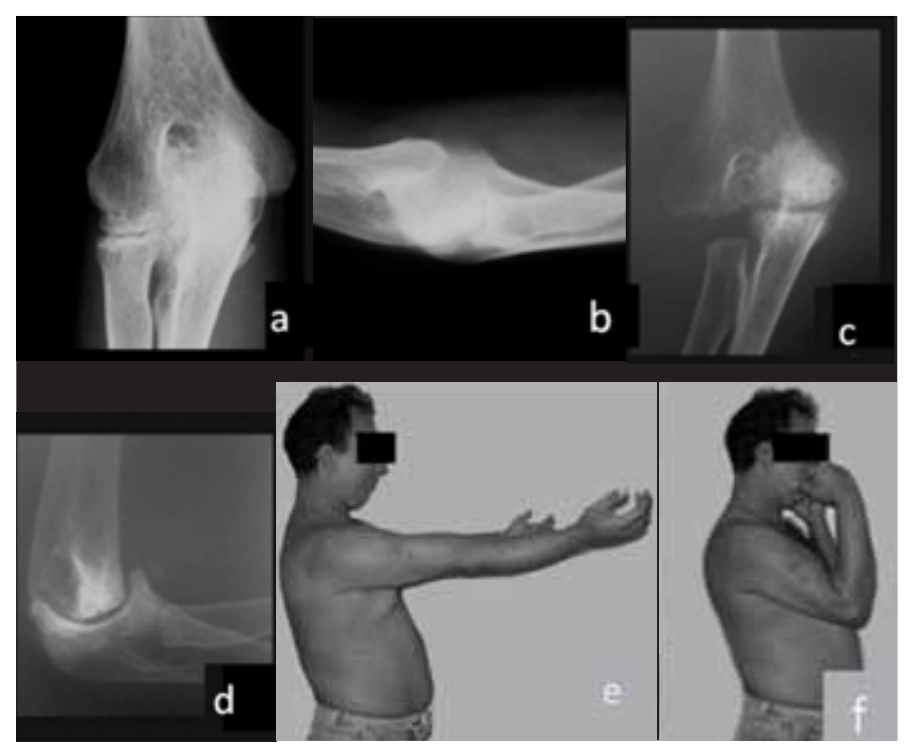

Figura 4 - Caso 4 - Imagens radiográficas de frente e perfil do cotovelo afetado no pré-operatório $(a, b)$ e no pós de um ano $(c, d)$. Mobilidade atual do paciente em extensão (e) e flexão máxima (f)

sidual em varo e valgo, citada por Nolla et al ${ }^{(10)}$ como um limitante importante das atividades dos pacientes, ela esteve presente, em menor ou maior grau, em todos os casos, com exceção do caso 5 , onde houve perda gradativa de movimento e hoje o cotovelo permanece praticamente anquilosado. Os pacientes foram treinados com terapia ocupacional a adaptar-se a esse tipo de instabilidade, modificando o modo de usar o membro superior em certas posições.

Os resultados da cirurgia foram avaliados de acordo com os critérios da AMA modificados por Bruce et $a^{(15)}$, diferentemente dos trabalhos de Cheng e Morrey ${ }^{(3)}$, Pignatti et $a^{(1)}$ e Nolla et $a l^{(10)}$, que utilizam os de MEPS (Mayo

\section{REFERÊNCIAS}

1. Pignatti G, Ferrari D, Tigani D, Scardovi M, Trentanti $P$, Trentani $F$, et al. II trattamento delle rigidità post-traumatiche del gomito. Chir Organi Mov. 2000;85(4):381-7.

2. Ljung P, Jonsson K, Larsson K, Rydholm U. Interposition arthroplasty of the elbow with rheumatoid arthritis. J Shoulder Elbow Surg. 1996;5(2 Pt 1):81-5.

3. Cheng SL, Morrey BF. Treatment of mobile, painful arthritic elbow by distraction interposition arthroplasty. J Bone Joint Surg Br. 2000;82(2):233-8.

4. Morrey BF, Askew LJ, Chao EY. A biomechanical study of normal functional elbow motion. J Bone Joint Surg Am. 1981;63(6):872-7.

5. Correia LS, Faria AP, Vieira LAG, Benegas E, Ferreira Neto AA, Zoppi Filho A. Artroplastia de ressecção na osteoartrose do cotovelo secundária à artrite reumatóide. Rev Bras Ortop. 2005;40(6):305-15.

6. Volkov MV, Oganesian OV. Restoration of function in the knee and elbow with a hingedistractor apparatus. J Bone Join Surg Am. 1975;57(5):591-600.

7. Wright PE 2nd, Sisk DT. Artroplastia de ombro e cotovelo. In: Crenshaw AH. Cirurgia ortopedica de Campbell. São Paulo: Manole; 1996. p.667-716.

8. Wright PE, Steward MJ. Fascial arthroplasty of the elbow. In: Morrey BF. The elbow and it's disorders. Philadelphia: Saunders; 1985. p.530-8.

9. Morrey BF. Distraction arthroplasty. Clin Orthop Relat Res. 1993;(293):46-54.

10. Nolla J, Ring D, Calderon SL, Jupiter JB. Interposition arthroplasty of the elbow with hinged external fixation for post-traumatic arthritis. J Shoulder Elbow Surg. 2008;17(3):459-64.

11. Deland JT, Garg A, Walker PS. Biomechanical basis for elbow hinge-distractor design. Clin Orthop Relat Res. 1987;(215): 303-12
Elbow Performance Score). Acreditamos que o primeiro método avalie melhor a função do membro superior.

Em todos os casos, com exceção do 5, verificamos aumento do índice de Bruce pré $(43,5)$ em relação ao pós-operatório $(88,2)$, demonstrando a eficiência do método para os casos de rigidez do cotovelo.

No Caso 5, a paciente tem artrite reumatoide juvenil (ARJ) e apresentou perda progressiva do arco de movimento do cotovelo após a retirada do fixador externo, retornando a graus de amplitude articular semelhantes aos encontrados no período pré-operatório. Acreditamos que esse insucesso pode ter sido causado pela falta de controle da doença de base (ARJ), que continuou em atividade, constatada por exames de sangue realizados recentemente. A paciente é acompanhada regularmente pela reumatologia do hospital, mas a doença parece ser de difícil controle.

Outro fator que colaborou para o insucesso do tratamento foi o fato de utilizarmos um único tamanho de fixador externo; pareceu-nos que, ao final da cirurgia, ele ficou muito grande para essa paciente: os pinos não ficaram na posição ideal, isto é, não conseguimos fazer o fulcro de movimento de flexoextensão do fixador no centro de rotação do cotovelo ${ }^{(11)}$. No período pós-operatório imediato, ainda com a paciente sob efeito anestésico, já não havia movimento completo da articulação, diferentemente de todos os outros casos.

\section{CONCLUSÃO}

O tratamento do cotovelo rígido pela técnica da artroplastia de interposição com fascia lata, associado ao uso de fixador externo dinâmico, mostrou-se, no presente estudo, uma alternativa viável no aumento da mobilidade articular em quatro de cinco pacientes assim tratados.
12. Morrey BF, An KN. Functional anatomy of the ligaments of the elbow. Clin Orthop Relat Res. 1985;(201):81-90.

13. Madey SM, Bottlang M, Steyers CM, Marsh JL, Brown TD. Hinged external fixation of the elbow: optimal axis alignment to minimize motion resistance. J Orthop Trauma. 2000;14(1):41-7.

14. Froimson AI. Facial interposition arthroplasty of the elbow. in the elbow. In: Morrey BF, editor. Master techniques in orthopeadic surgery. 2nd ed. New York: Raven Press; 1994.

15. Bruce HE, Harvey JP, Wilson JC Jr. Monteggia fractures. J Bone Joint Surg Am. 1974;56(8):1563-76.

16. Pöll RG, Rozing PM. Use of the Souter-Strathclyde total elbow arthroplasty in patients who have rheumatoid arthritis. J Bone Joint Surg Am. 1991;73(8):1227-33.

17. Lyall HA, Cohen B, Clatworthy M, Constant CR. Results of the Souter-Strathclyde total elbow arthroplasty in patients with rheumatoid arthritis: a preliminary report. J Arthroplasty. 1994; 9(3):279-84.

18. Sjoden GO, Lundberg A, Blomgren GA. Late results of the Souter-Strathclyde total elbow prosthesis in rheumatoid arthritis: $6 / 19$ implants loose after 5 years. Acta Orthop Scand. 1995;66(5):391-4.

19. Cobb TK, Morrey BF. Use of distraction arthroplasty in unstable fracture dislocations of the elbow. Clin Orthop Relat Res.1995;(312):201-10.

20. Vancabeke M, Lamraski G, Berthe JV, Coessens BC. One-stage elbow interposition arthroplasty with a fasciocutaneous distally planned lateral arm flap. Acta Orthop Belg. 2002;68(4):392-5.

21. Ring D, Hotchkiss RN, Guss D, Jupiter JB. Hinged elbow external fixation for severe elbow contracture. J Bone Joint Surg Am. 2005;87(6):1293-6. 